\title{
Apoptin sensitizes radiation-induced cell death via classic mitochondrial, caspase and p53-dependent signaling in HepG2 cells
}

\author{
SU-XIA HAN ${ }^{1}$, QING ZHU ${ }^{1}$, JIN-LU MA ${ }^{1}$, YI LV ${ }^{1}$, JING ZHAO ${ }^{1}$, \\ CHEN HUANG $^{2}$, XI JIA ${ }^{1}$, WEI OU ${ }^{1}$ and HONG-TAO GUO ${ }^{1}$ \\ ${ }^{1}$ Department of Oncology, The First Affiliated Hospital of Xi'an Jiaotong University College of Medicine; \\ ${ }^{2}$ Centre Laboratory, Xi'an Jiaotong University College of Medicine, Xi'an 710061, P.R. China
}

Received August 11, 2010; Accepted October 18, 2010

DOI: $10.3892 / \mathrm{mmr} .2010 .391$

\begin{abstract}
Resistance or insensitivity to radiation therapy is one of the hallmarks of hepatocellular carcinoma. Sensitizing radioresistant cancer by combining radiation with other therapeutics to induce apoptosis has been widely investigated. Our previous study showed that chicken anaemia virus-derived apoptin protein induced the apoptosis of hepatic carcinoma HepG2 cells. In the present study, we demonstrated that apoptin sensitizes cells to radiation-induced apoptosis using a lentivirus-apoptin expression system in hepatic carcinoma HepG2 cells. Combination therapy with radiation and apoptin dramatically induced mitochondrial cytochrome c release and the cleavage of caspases $-9,-3$ and -7 . Our findings are also the first to show that the combination of radiation and apoptin up-regulates p53 expression. Thus, apoptin treatment represents a potential method for enhancing the effectiveness of radiotherapy in poorly responding hepatocellular carcinoma.
\end{abstract}

\section{Introduction}

Hepatocellular carcinoma is the sixth most common cancer worldwide and the third most common cause of cancer-related death, particularly in developing countries. Hepatocellular carcinoma patients have fairly poor outcomes, with only a $3-5 \%$ survival rate at the 5-year benchmark (1). Major treatment options for patients include liver resection and subsequent liver transplantation; however, these are not possible in many patients (2). Reasons include poor liver function, macrovascular tumor invasion and extended wait times for donor organs (3).

Correspondence to: Dr Su-Xia Han, Department of Oncology, The First Affiliated Hospital of Xi'an Jiaotong University College of Medicine, Xi'an 710061, P.R. China

E-mail: hsummer22099@yahoo.cn

Abbreviations: RT, radiation therapy; MOI, multiplicity of infection; Lenti-Ap, lentivirus-apoptin; Lenti-C, lentivirus GFP control

Key words: apoptin, hepatocellular carcinoma, radiation, apoptosis
Radiation therapy (RT) has historically played a minor role in the management of unresectable tumors, since these cancer cells are not sensitive to radiation. They also present a clinical challenge, since RT dose-escalation is not well-tolerated by the liver (4). Researchers are therefore examining combination treatments using novel strategies that may allow for a decreased dosage and enhanced sensitivity to radiation.

Apoptin has increasingly been shown to be of value in specifically targeting and eliminating cancer cells (5). This protein was first isolated from the chicken anemia virus, and its potential anti-cancer effects appear to be tumor specific; transformed cells are specifically targeted, while primary and non-transformed cells are spared (6). The apoptotic activity of apoptin is suggested to occur via a p53-independent, Bcl-2enhanced mechanism $(7,8)$.

Our previous study showed that secretory transactivating transcription apoptin fusion protein induces cell apoptosis in hepatocellular carcinoma HepG2 cells $(9,10)$. In the present study, we sought to explore the anti-tumor effect of a combination of RT and apoptin gene therapy in radio-resistant hepatocellular carcinoma HepG2 cells, and to further investigate the mechanisms of apoptosis. The results indicated that combination treatment with RT and apoptin has a beneficial effect on the apoptosis of radio-resistant hepatocellular carcinoma HepG2 cells through mitochondrial proteins, caspase activation and $\mathrm{p} 53$-dependent signaling.

\section{Materials and methods}

Cells and reagents. The human hepatocellular carcinoma cell line HepG2 was obtained from the American Type Culture Collection (Manassas, VA, USA). Cells were cultured in Dulbecco's modified Eagle's medium (DMEM; Gibco-BRL, Grand Island, NY, USA) with $10 \%$ fetal bovine serum (Hyclone, Logan, UT, USA) and 1\% penicillin-streptomycin (Gibco-BRL) under standard culture conditions $\left(37^{\circ} \mathrm{C}, 95 \%\right.$ humidified air and 5\% $\mathrm{CO}_{2}$ ). Anti-Bcl-2, anti-Bax and anti-Bak primary antibodies were from Upstate Biotechnologies (Lake Placid, NY, USA). Antibodies which specifically recognize p53 and cleaved-caspases as well as peroxidase-conjugated anti-rabbit secondary antibody were from Cell Signaling 
Technology (Beverly, MA, USA). Anti-Bid antibody was from R\&D Systems (Minneapolis, MN, USA). The ECL system was from Amersham (Piscataway, NJ, USA). Other chemicals were obtained in their commercially available highest purity grade.

Generation of recombinant lentivirus and transfection into HepG2 cells. Lentiviral pSMPUW-IRES-GFP vector, packaging vector $\mathrm{pCgpV}$ and envelope vector pCMV-Eco (Cell Biolab Inc., USA) were co-transfected into 293T cells. The viral particles were collected $48 \mathrm{~h}$ later. In order to investigate apoptotic effects and related proteins, HepG2 cells were infected with viral supernatants in the presence of polybrene $(6 \mu \mathrm{g} / \mathrm{ml})$ for $24 \mathrm{~h}$ at multiplicities of infection (MOIs) of 1 , 3,5 or 10 . The transfected HepG2 cells were sub-cultured for 1-2 days after transfection for further study.

Radiation treatment. HepG2 cells were grown to $\sim 80 \%$ confluence in DMEM under standard culture conditions. Irradiated cells were transfected with various recombinant lentiviruses at the indicated MOIs of lentivirus-apoptin (Lenti-Ap), or with a GFP lentivirus control (Lenti-C). Briefly, fresh medium was added to each well and the wells were incubated for $24 \mathrm{~h}$. One day after viral infection, cells were irradiated at $37^{\circ} \mathrm{C}$ using a $6 \mathrm{MV}$ linear accelerator. Doses were administered in the fraction of 1-10 Gy (17). After irradiation, the dishes were immediately returned to the incubator for a further $24 \mathrm{~h}$ of culture.

Apoptosis assay. HepG2 cells prepared as above were collected at the desired time periods and stained with annexin $\mathrm{V}$ and PI (Molecular Probes) following the manufacturer's protocol. Early apoptotic cells were analyzed immediately by flow cytometry as described previously (9).

Preparation of mitochondrial and cytosolic fractions. The preparation of mitochondrial and cytosolic lysates was carried out as described previously (18). Briefly, HepG2 cells were lysed in lysate buffer (20 mM Hepes, pH 7.5, $10 \mathrm{mM} \mathrm{KCl}$, $1.9 \mathrm{mM} \mathrm{MgCl}_{2}, 1 \mathrm{mM}$ EGTA and $1 \mathrm{mM}$ EDTA) with protease inhibitors on ice for $20 \mathrm{~min}$. The cytosolic supernatant proteins were centrifuged at $14,000 \mathrm{x}$ g for $30 \mathrm{~min}$, following centrifugation at $1,000 \mathrm{x} \mathrm{g}$ for $5 \mathrm{~min}$; the pellets were then suspended in mitochondrial lysate buffer $(150 \mathrm{mM} \mathrm{NaCl}$, $50 \mathrm{mM}$ Tris- $\mathrm{HCl}, \mathrm{pH} 7.4,1 \% \mathrm{NP}-40,0.25 \%$ sodium deoxycholate and $1 \mathrm{mM}$ EDTA) with protease inhibitors on ice for $30 \mathrm{~min}$ to collect mitochondrial proteins.

Western blotting. Infected cells were collected at $48 \mathrm{~h}$ postinfection and lysed in SDS sample buffer, and proteins were separated using SDS-PAGE. Separated proteins were transferred onto nitrocellulose membranes. Western blotting was carried out as described previously (19).

Statistical analysis. All experiments were repeated at least in triplicate. The data were analyzed using the SPSS statistical package (ver. 10.0; Chicago, IL, USA). For all measurements, when required, the t-test was employed to assess the statistical significance of differences between the treatment groups. p-values $<0.05$ were considered significant.
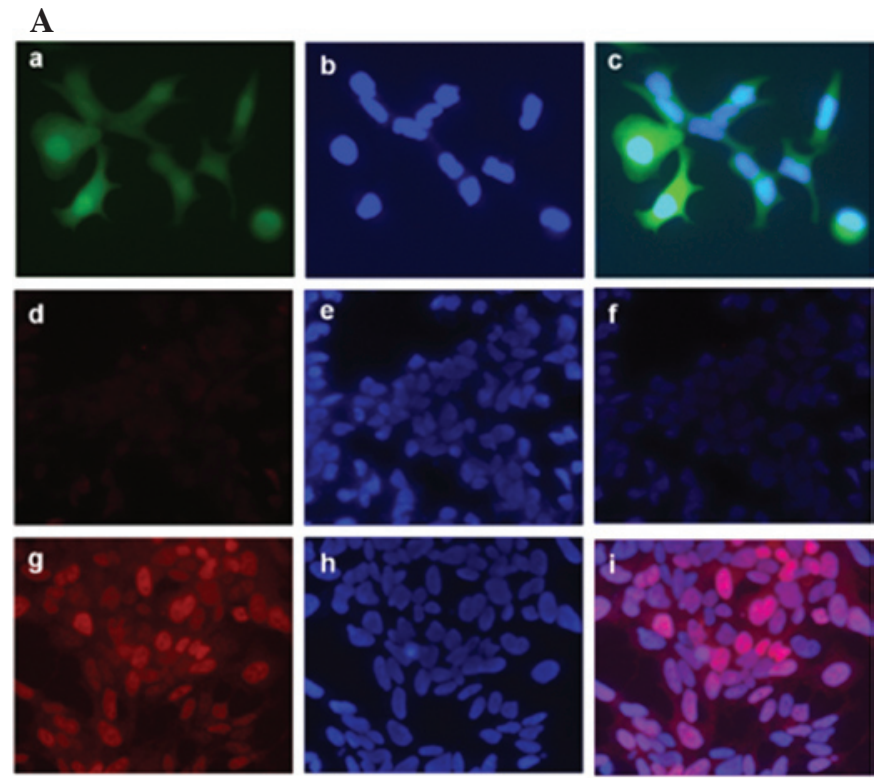

$\mathbf{B}$

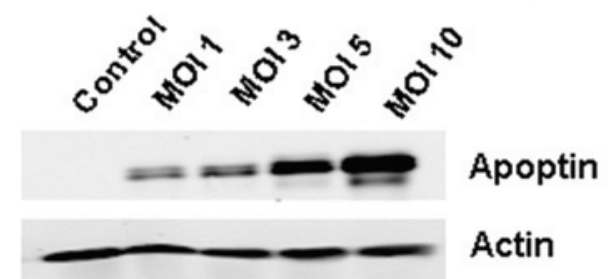

Figure 1. Lentivirus-mediated apoptin expression in HepG2 cells. (A) Cells were infected with the recombinant lentivirus apoptin or GFP lentivirus control for $24 \mathrm{~h}$ and then fixed at room temperature for $15 \mathrm{~min}$. Apoptin detected by anti-apoptin Alexa Fluor 594 conjugated secondary antibodies is shown in red and cell nuclei stained by DAPI in blue. Apoptin protein was mainly located in the nucleus. a, lentivirus-GFP GFP; $b$, lentivirus-GFP DAPI; $c$, lentivirus-GFP merge; d, lentivirus-GFP apoptin; e, lentivirus-GFP DAPI; $\mathrm{f}$, lentivirus-GFP merge; g, lentivirus-apoptin apoptin; h, lentivirus-apoptin DAPI; i, lentivirus-apoptin merge. Magnification, x400. (B) Apoptin protein expression following lentivirus infection at the indicated MOIs confirmed by Western blotting.

\section{Results}

Lentivirus-mediated apoptin expression in HepG2 cells. The HepG2 cells were first modulated through a lentivirus-apoptin expression system. To confirm the overexpression of apoptin, immunofluorescence staining was performed $24 \mathrm{~h}$ after transfection. Apoptin expression was significantly increased in cells transfected with Lenti-Ap compared to Lenti-C $(\mathrm{p}<0.05)$, and apoptin was mainly concentrated in the nucleus, not the cytosol. This is consistent with the results of our previous study (Fig. 1A) (9), indicating that a lentivirus-apoptin expression system is capable of dramatically up-regulating apoptin expression in HepG2 cells.

Apoptin expression in HepG2 cells was further confirmed by Western blot analysis (Fig. 1B). Apoptin expression was up-regulated in a dose-dependent manner following $24 \mathrm{~h}$ of infection with the various concentrations of lentivirus particles (Fig. 1B).

Apoptin enhances radiation-mediated apoptosis of HepG2 cells. Radiation and lentivirus-mediated cell apoptosis was assessed at various doses and different times in the HepG2 
A

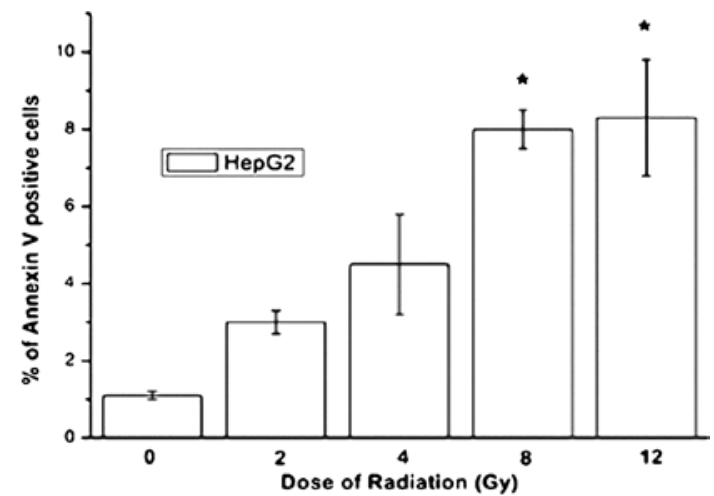

B

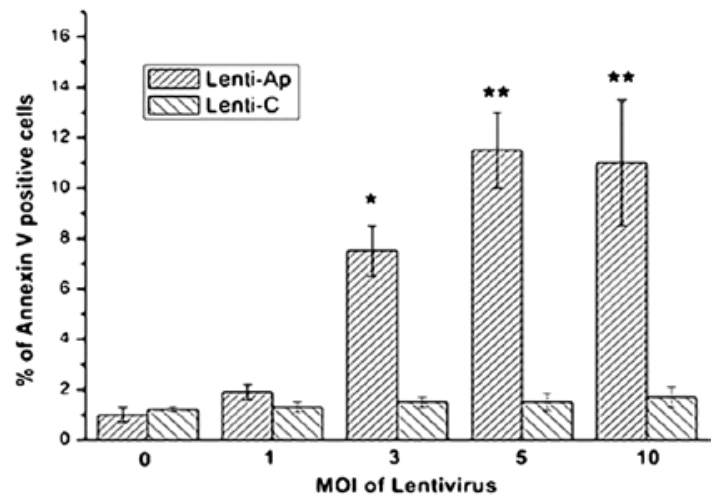

C

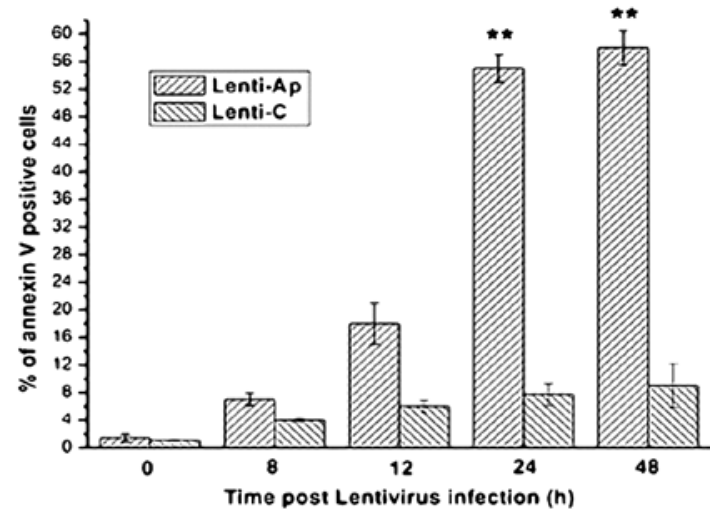

Figure 2. Dose- and time-dependent apoptotic death induced by apoptin and radiation in HepG2 cells. (A) Cells were exposed to various doses of radiation ( $0-12 \mathrm{~Gy})$, then harvested after $6 \mathrm{~h}$ and stained with annexin $\mathrm{V}$ and PI, followed by flow cytometric analysis. (B) Cells were infected with the indicated MOIs of lentivirus particles (0-10 MOI) and incubated at $37^{\circ} \mathrm{C}$ for $24 \mathrm{~h}$, then harvested and stained with annexin $\mathrm{V}$ and PI, followed by flow cytometric analysis. (C) Cells were exposed to $8 \mathrm{~Gy}$ of radiation and treated with lentivirus at an MOI of 5 for the indicated time periods. At each time point, cells were harvested and stained with annexin V and PI, followed by flow cytometric analysis. Quantification of apoptosis was conducted using only annexin V-positive cells. In each case, the data shown are the means \pm SE of three samples. ${ }^{*} \mathrm{p}<0.05 ;{ }^{* *} \mathrm{p}<0.01$ compared to the control.

cells. Irradiation induced apoptotic cell death in a dosedependent manner in the HepG2 cells. Maximal apoptotic effects were observed at 8 Gy of radiation $(\mathrm{p}<0.05)$, from a range of 2-12 Gy. Results were obtained using an apoptosis assay with annexin V/PI staining and FACS analysis (Fig. 2A).

Following apoptin virus infection, apoptin-mediated apoptosis was observed at a MOI of 5, with the maximal response occurring after $24 \mathrm{~h}$ of incubation. The control cells exhibited a large viable cell population with not much staining of apop-

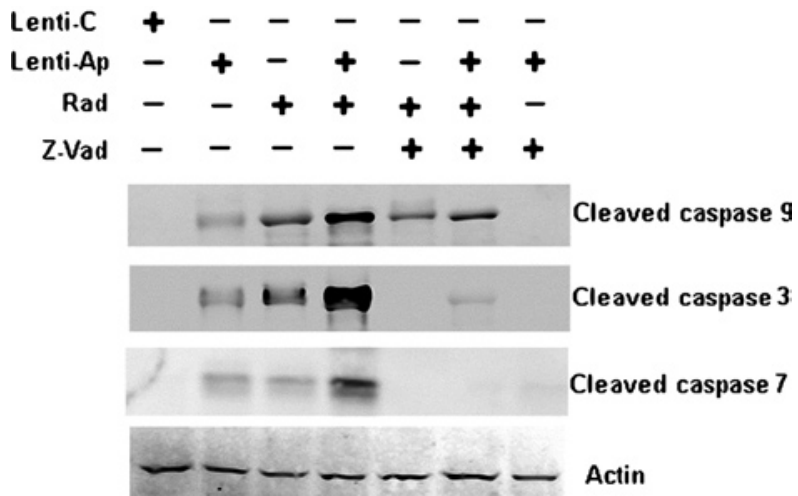

Figure 3. Effect of apoptin on radiation-induced caspases $-9,-3$ and -7 cleavage in HepG2 cells. Sub-confluent HepG2 cell cultures in 100-mm dishes were exposed to $8 \mathrm{~Gy}$ of radiation alone and/or with exposure to lentivirus particles at an MOI of 5 . Cleaved caspases $-9,-3$ and -7 were detected by Western blotting.

totic cells, even following infection with GFP control virus at an MOI of 10 (Fig. 2B).

To determine the time-dependent effects of combination treatment with apoptin and radiation, HepG2 cells were treated with apoptin virus particles at an MOI of 5 for different time periods and exposed to $8 \mathrm{~Gy}$ of radiation. Compared to cells infected with GFP control virus alone, a significant additional shift from live cells to an early apoptotic cell population was observed (Fig. 2C). A significant increase in apoptosis was observed after $24 \mathrm{~h}$ of treatment with apoptin virus in combination with radiation exposure $(\mathrm{p}<0.01)$ (Fig. 2C).

Overall, these findings suggest that post-treatment with apoptin virus sensitizes HepG2 cells to radiation-induced cell apoptosis.

Apoptin enhances radiation-induced caspase activation in HepG2 cells. Experimental treatments were conducted to assess apoptin-mediated caspase activation in HepG2 cells exposed to either radiation or apoptin virus alone, or to apoptin virus and radiation in combination. Some cells were exposed to the pan-caspase inhibitor Z-VAD-FMK following radiation and virus treatment. For Western blotting, a specific antibody was used to recognize only activated caspase-9 (the cleaved product of procaspase-9).

As shown in Fig. 3, treatment with apoptin virus in combination with radiation exposure further increased the level of cleaved caspase-9 compared to radiation or apoptin exposure alone. However, this increase in cleaved caspase-9 was suppressed when cells were treated with the pan-caspase inhibitor Z-VAD-FMK after irradiation.

Under the same conditions, the activation of caspase- 3 and -7 was assessed. High levels of cleaved caspase- 3 and -7 were observed after radiation exposure, consistent with the caspase- 9 results for apoptosis. Cleaved caspase-3 and -7 levels additionally increased when cells were post-treated with apoptin virus. When cells were treated with the pan-caspase inhibitor Z-VAD-FMK after radiation exposure, cleaved caspase-7 and -3 were completely inhibited; however, cleaved caspase-3 was not as inhibited as with treatment with apoptin virus at an MOI of 5 and 8 Gy of radiation.

These findings suggest that caspase activation plays a major role in radiation-induced apoptosis. Post-treatment with 


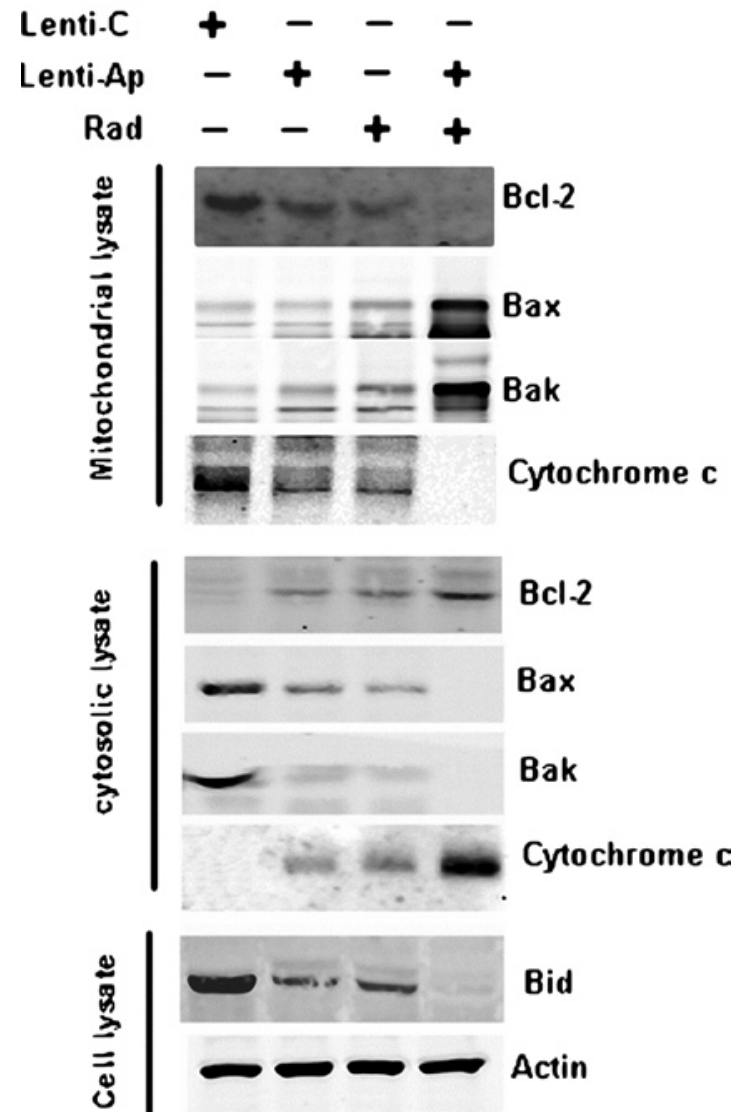

Figure 4. Effect of apoptin and/or radiation on Bcl-2 family proteins and cytochrome $\mathrm{c}$ release in HepG2 cells. HepG2 cells were exposed to 8 Gy radiation alone and/or with apoptin virus treatment at an MOI of 5. Mitochondrial and cytosolic fractions were measured by Western blotting. Upper panel: Bcl-2, Bax, Bak and cytochrome c in mitochondria; middle panel: Bcl-2, Bax, Bak and cytochrome $\mathrm{c}$ in cytosolic fractions; lower panel: BID.

apoptin significantly enhances the radiation-induced apoptotic death of HepG2 cells.

Apoptin modulates radiation-induced translocation of $\mathrm{Bcl}-2$ family members and cytochrome c release in HepG2 cells. Mitochondrial, cytosolic and total proteins were prepared in order to determine the expression of Bcl-2 family members in apoptin and radiation-mediated apoptosis. Protein levels and the translocation of Bcl-2 family members were assessed.

Fig. 4 shows a significant decrease in Bcl-2 levels in mitochondrial proteins treated with apoptin virus and radiation compared to treatment with radiation alone. In the cytosolic fraction, apoptin virus treatment in combination with RT significantly increased the level of Bcl-2 compared to treatment with apoptin or radiation alone. Overall, virus treatment after radiation exposure decreased the total level of Bcl-2.

Conversely, radiation both with or without apoptin had a significant effect on the levels of pro-apoptotic Bax and Bak in mitochondria, whereas in the cytosol Bax and Bak levels were down-regulated by radiation in combination with apoptin treatment compared to treatment with radiation alone. Taken together, these results suggest that treatment with apoption in combination with radiation exposure is mediated by a decreased level of $\mathrm{Bcl}-2$ in mitochondria and of total $\mathrm{Bcl}-2$, Bax and Bak proteins in the cytosol.

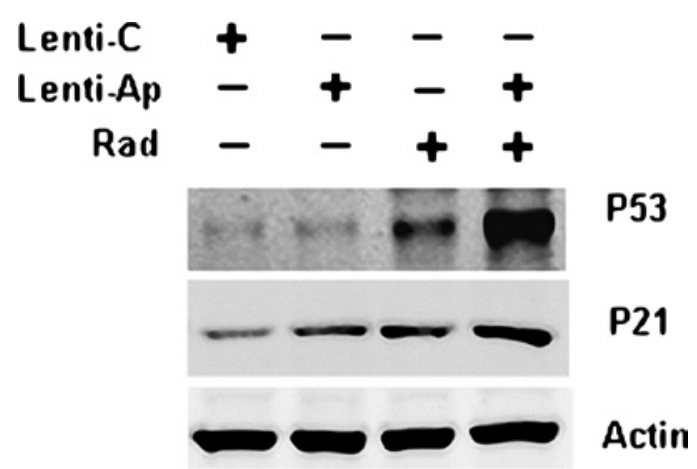

Figure 5. Expression of p53 and p21 induced by apoptin and/or radiation in HepG2 cells. HepG2 cells were exposed to $8 \mathrm{~Gy}$ of radiation alone and/or with apoptin virus treatment at an MOI of 5. Levels of p53 and p21 were evaluated by Western blot analysis using respective antibodies against p53 and p21.

Radiation in combination with apoptin virus treatment decreased the level of mitochondrial cytochrome c; however, the level of cytosolic cytochrome $\mathrm{c}$ was increased as compared to control virus-treated or parental cells. BID cleavage was also assessed in the whole cell lysates. Consistent with Bcl-2 expression in mitochondria, radiation in combination with apoptin virus treatment dramatically decreased the level of total BID. Decreased BID levels suggest that BID cleavage may be another molecular target of combination treatment with apoptin and radiation.

The results suggest that apoptin modulates the protein levels and also alters the translocation of $\mathrm{Bcl}-2$ family proteins in response to radiation. This is thought to contribute to HepG2 cell apoptosis.

Apoptin modulates radiation-induced cell apoptosis through p53 pathways in HepG2 cells. A previous study showed that apoptin alone induced p53-independent cancer cell apoptosis (8). In the present study, we sought to explore the role of the p53 pathway in apoptin-induced apoptosis following radiation in HepG2 cells. The combination of apoptin and radiation exposure increased the level of p53 protein and its downstream p21 expression compared to the low basal levels of p53 and p21 observed in control cells (Fig. 5). This suggests that cell apoptosis induced by the combination of apoptin virus treatment and radiation exposure, rather than apoptin virus treatment or radiation exposure alone, is p53 pathwaydependent.

\section{Discussion}

Resistance or insensitivity to radiotherapy in hepatocarcinoma cells is due to acquired deficiency in the apoptotic program, which limits the application of radiation in the clinical setting (11). Researchers in oncology are attempting to develop novel strategies to sensitize such tumor cells to radiation. In the present study, we generated a recombinant lentivirus vector expressing apoptin and investigated its tumor-specific apoptosis-inducing activity following radiation in human radiation-resistant hepatic carcinoma HepG2 cells. Our findings showed that the tumor-selective apoptosis inducing protein apoptin has a clear additive cytotoxic effect on hepatic carcinoma HepG2 cells treated by radiation, which triggered 
classic apoptosis pathways through both mitochondrial and caspase-dependent processes. We also showed for the first time that apoptin sensitized the cells to radiation-mediated apoptosis through a p53-dependent pathway. This finding is of potential value in a clinical setting, particularly for radiationresistant hepatic carcinoma cells.

In our previous study, we demonstrated that apoptin promoted HepG2 cell apoptosis by a secretory transactivating transcription-apoptin fusion protein system (9). In the present study, concurrent treatment of HepG2 radio-resistant cells with radiation and apoptin lentivirus was shown to further increase apoptotic cell death compared to either treatment alone, suggesting that apoptin sensitizes radio-resistant HepG2 cells to radiation. This assumption is strengthened by the findings that radiation alone failed to activate the pro-apoptotic caspases, mitochondrial cytochrome $\mathrm{c}$ release and the level of p53 in the radio-resistant HepG2 cells. However, this activation did occur following radiation in combination with apoptin treatment. These results suggest that, in contrast to radiation, apoptin activates the apoptotic machinery in HepG2 cells through classical mitochondrial, caspase and p53-dependent pathways.

Apoptin induces cell apoptosis in the absence of the tumor suppressor p53 $(8,12)$. In the present study, we first demonstrated that radiation alone slightly activated p53, but significantly increased the level of p53 in combination with apoptin, indicating that apoptin sensitized radiation-mediated p53 expression. Such therapy may be useful for the treatment of tumors that have lost p53 and are therefore resistant to many forms of anti-cancer agents, including radiotherapy (13).

Activation of caspases is very important in apoptosis (14), playing an essential role in the cleavage of DNA, which in turn leads to apoptosis (15). Of the many upstream and downstream caspases, in our present study we examined the involvement of caspases $-9,-3$ and -7 in combination with apoptin and radiation-mediated apoptosis in human hepatic carcinoma HepG2 cells. Our results showed apoptin and radiation-induced synergetic apoptosis, indicating that the caspase pathways are partially involved in apoptin and radiation-mediated cell apoptosis.

In addition, a number of previous studies have shown that apoptin induces apoptosis in cells expressing anti-apoptotic proteins of the Bcl-2 family $(8,12)$. Both apoptotic and antiapoptotic proteins are found in the Bcl-2 family, and these have been reported to play a key role in the mitochondrial apoptotic pathway. Bcl-2 has an anti-apoptotic role, whereas Bax and Bak have pro-apoptotic roles. Homo- and/or heterodimerization of Bcl-2 family proteins control the cell apoptotic or survival pathways. Homodimers of Bcl-2 block cytochrome c release from mitochondria, thereby preventing the initiation of caspase activation. However, heterodimers of Bax or Bak with Bcl-2 activate apoptosis (16). In our study, we demonstrated that apoptin altered the translocation of Bcl-2 family proteins in response to radiation stress, which further triggered the apoptotic effect in HepG2 cells.

In conclusion, the present study demonstrated using a lentivirus-apoptin expression system that apoptin sensitizes radiation-mediated cell apoptosis through classic mitochondrial, caspase and p53-dependent pathways in hepatic carcinoma HepG2 cells. Based on our present and previous study, apoptin, which selectively targets cancer cells, but spares normal cells, represents a novel anti-cancer modality and may have great clinical significance. Thus, gene therapy with apoptin combined with traditional RT may offer unique advantages over current therapies for radiation-insensitive hepatocellular carcinoma.

\section{Acknowledgements}

This study was supported by the National Natural Science Foundation of China (no. 81071692) and the Shaanxi Provincial Natural Science Foundation (no. 2010JM4021).

\section{References}

1. Parkin DM, Bray F, Ferlay J and Pisani P: Global cancer statistics, 2002. CA Cancer J Clin 55: 74-108, 2005.

2. Stone MJ, Klintmalm GB, Polter D, et al: Neoadjuvant chemotherapy and liver transplantation for hepatocellular carcinoma: a pilot study in 20 patients. Gastroenterology 104: 196-202, 1993.

3. Llovet JM, Schwartz M, Fuster J and Bruix J: Expanded criteria for hepatocellular carcinoma through down-staging prior to liver transplantation: not yet there. Semin Liver Dis 26: 248-253, 2006.

4. Emami B, Lyman J, Brown A, et al: Tolerance of normal tissue to therapeutic radiation. Int J Radiat Oncol Biol Phys 21: 109-122, 1991.

5. Noteborn MH: Proteins selectively killing tumor cells. Eur J Pharmacol 625: 165-173, 2009.

6. Noteborn MH: Apoptin acts as a tumor-specific killer: potentials for an anti-tumor therapy. Cell Mol Biol 51: 49-60, 2005.

7. Burek M, Maddika S, Burek CJ, Daniel PT, Schulze-Osthoff K and Los M: Apoptin-induced cell death is modulated by Bcl-2 family members and is Apaf-1 dependent. Oncogene 25: 2213-2222, 2006.

8. Schoop RA, Kooistra K, De Jong RJ and Noteborn MH: Bcl-xL inhibits p53- but not apoptin-induced apoptosis in head and neck squamous cell carcinoma cell line. Int J Cancer 109: 38-42, 2004.

9. Han SX, Ma JL, Lv Y, Huang C, Liang HH and Duan KM: Secretory transactivating transcription-apoptin fusion protein induces apoptosis in hepatocellular carcinoma HepG2 cells. World J Gastroenterol 14: 3642-3649, 2008.

10. Han SX, Ma JL, Lu Y, Huang C and Duan KM: SP-TAT-apoptin induces G1 arrest in HepG2 cells. Xi Bao Yu Fen Zi Mian Yi Xue Za Zhi 24: 864-866, 2008.

11. Fulda S and Debatin KM: Targeting apoptosis pathways in cancer therapy. Curr Cancer Drug Targets 4: 569-576, 2004

12. Danen-van Oorschot AA, van der Eb AJ and Noteborn MH: BCL-2 stimulates apoptin-induced apoptosis. Adv Exp Med Biol 457: 245-249, 1999.

13. Heilman DW, Teodoro JG and Green MR: Apoptin nucleocytoplasmic shuttling is required for cell type-specific localization, apoptosis, and recruitment of the anaphase-promoting complex/ cyclosome to PML bodies. J Virol 80: 7535-7545, 2006.

14. Zheng TS and Flavell RA: Apoptosis. All's well that ends dead. Nature 400: 410-411, 1999.

15. Thornberry NA and Lazebnik Y: Caspases: enemies within. Science 281: 1312-1316, 1998.

16. Patel MP, Masood A, Patel PS and Chanan-Khan AA: Targeting the Bcl-2. Curr Opin Oncol 21: 516-523, 2009.

17. Tang WY, Chau SP, Tsang WP, Kong SK and Kwok TT: The role of Raf-1 in radiation resistance of human hepatocellular carcinoma Hep G2 cells. Oncol Rep 12: 1349-1354, 2004.

18. Singh RP, Dhanalakshmi S, Tyagi AK, Chan DC, Agarwal C and Agarwal R: Dietary feeding of silibinin inhibits advance human prostate carcinoma growth in athymic nude mice and increases plasma insulin-like growth factor-binding protein-3 levels. Cancer Res 62: 3063-3069, 2002.

19. Li X, Jin N, Mi Z, Lian H, Sun L, Li X and Zheng H: Antitumor effects of a recombinant fowlpox virus expressing apoptin in vivo and in vitro. Int J Cancer 119: 2948-2957, 2006. 